\title{
Relación entre sobrecarga y competencias del cuidar en cuidadores informales de personas con enfermedades crónicas
}

The relationship between overload and caregiving competencies in informal caregivers of people with chronic diseases

Relação entre sobrecarga e competências do cuidar em cuidadores informais de pessoas com doenças crônicas

Julia Elena Del Ángel-García, Enf.1 (®, Rodrigo César León Hernández, Psiq., PhD. ${ }^{2}$ (๑), Gregorio Méndez Santos, Enf. ${ }^{3}$, Isabel Peñarrieta de Córdova, Enf., PhD. ${ }^{4}$ (), Florabel Flores-Barrios, Enf., MSc. 5 ๑.

1. Licenciada en Enfermería, Facultad de Enfermería de Tampico, Universidad Autónoma de Tamaulipas, México.

2. Doctor en Psicología de la Salud, Consejo Nacional de Ciencia y Tecnología, México.

3. Enfermero, Pasante de Servicio Social, Facultad de Enfermería de Tampico, Universidad Autónoma de Tamaulipas, México.

4. Doctora en Salud Pública, Facultad de Enfermería de Tampico, Universidad Autónoma de Tamaulipas, México.

5. Maestra en Ciencias de Enfermería, Facultad de Enfermería de Tampico, Universidad Autónoma de Tamaulipas, México.

Correspondencia. Julia Elena Del Ángel García. Avenida Universidad con Wisconsin. Teléfono 8331852467. Tamaulipas, México Email. juliaelenadelangel@gmail.com

\section{INFORMACIÓN DEL ARTÍCULO:}

Artículo recibido: 01 de marzo 2020

Artículo aceptado: 10 de junio 2020

DOI: https://doi.org/10.29375/01237047.3878

Cómo citar. Del Ángel-García JE, León-Hernández RC, Méndez-Santos G, Peñarrieta-De Córdova I, Flores-Barrios F. Relación entre sobrecarga y competenciasdel cuidaren cuidadoresinformales de personascon enfermedades crónicas. MedUNAB. 2020;23(2): 233-241. doi: 10.29375/01237047.3878

\section{RESUMEN}

Introducción. El desarrollo de enfermedades crónicas y sus posibles complicaciones conllevan la necesidad de recibir cuidados de alguna persona del entorno social o familiar, quien se convierte en cuidador informal. Al desempeñar su rol, esta persona puede experimentar situaciones o características propias del cuidador que repercuten 
en las competencias del cuidar y sobrecarga, aspectos que podrían afectar la díada cuidador-cuidado. Objetivo. Identificar la relación entre competencias del cuidar y sobrecarga en cuidadores informales de personas con enfermedades crónicas. Metodología. Estudio transversal-correlacional, $\mathrm{n}=259$ cuidadores informales de personas con enfermedades crónicas, usuarios de dos hospitales generales de Tamaulipas, seleccionados por muestreo no probabilístico, intencional. Se aplicó una cédula de datos sociodemográficos, instrumento Zarit y CUIDAR. Para el análisis, se aplicó estadística descriptiva e inferencial. Resultados. Los cuidadores fueron mayormente mujeres, con parentesco de hija y esposa. Se detectó una correlación negativa y estadísticamente significativa ( $\mathrm{rs}=-.160, \mathrm{p}=0.01$ ) entre la sobrecarga y competencias del cuidar. Discusión. El perfil de los cuidadores de este estudio concuerda con la literatura internacional. La correlación entre las variables de investigación se ha documentado en escasos estudios, por lo que representa un avance de conocimiento en el ámbito de los cuidadores. Conclusión. Se evidenció una correlación que se puede interpretar de la siguiente manera: "a mayor sobrecarga, menor competencias del cuidar". Hallazgo que evidencia necesidades en conocimientos y habilidades para ejercer de forma óptima el rol de cuidador y por ende, tener la capacidad de afrontar situaciones de sobrecarga.

Palabras clave:

Cuidadores; Enfermedades Crónicas; Pacientes; Adulto; Diabetes Mellitus; Hipertensión.

\begin{abstract}
Introduction. When people develop chronic diseases and their possible complications, it leads to the need to receive care from someone in social or family contexts. This person becomes an informal caregiver. By playing this role, this person can experience situations or characteristics inherent to caregivers, which impact caregiving competencies and overload. These aspects could affect the caregiver-care receiver dyad. Objective. To identify the relationship between caregiving competencies and overload in informal caregivers of people with chronic diseases. Methods. A transversal, correlational study, $n=259$, on informal caregivers of people with chronic diseases, users of two general hospitals in Tamaulipas, selected through intentional non-probability sampling. A sociodemographic data form and the Zarit and CUIDAR instruments were used. Descriptive and inferential statistics were applied for the analysis. Results. Caregivers were mostly women, with kinship as daughters or wives. A negative, statistically significant correlation was detected ( $\mathrm{rs}=-.160, \mathrm{p}=0.01)$ between overload and caregiving competencies. Discussion. Caregivers' profiles in this study concur with international literature. The correlation between research variables has been documented in very few studies, reason why it represents progress in knowledge in the field of caregiving. Conclusion. A correlation that can be interpreted as follows was observed: "more overload implies less caregiving competencies." This finding demonstrates the need for knowledge and skills to optimally provide care and, therefore, have the capacity to face overload situations.
\end{abstract}

Keywords:

Caregivers; Chronic Diseases; Patients; Adults; Diabetes Mellitus; Hypertension.

\title{
RESUMO
}

Introdução. O desenvolvimento de doenças crônicas e suas possíveis complicações acarretam a necessidade de receber cuidados de alguém no ambiente social ou familiar, que se torna cuidador informal. Ao desempenhar seu papel, essa pessoa pode experimentar situações ou características próprias dos cuidadores que tange às competências para cuidar e sobrecarga, aspectos que podem afetar a díade cuidador-cuidado. Objetivo. Identificar a relação entre as competências do cuidar e a sobrecarga em cuidadores informais de pessoas com doenças crônicas. Métodos. Estudo transversal correlacional, $n=259$ cuidadores informais de pessoas com doenças crônicas, usuários de dois hospitais gerais de Tamaulipas, selecionados por amostragem intencional não probabilística. Foram aplicados informações sociodemográficas, escala Zarit e CUIDAR. Para a análise, aplicou-se estatística descritiva e inferencial. Resultados. Os cuidadores eram em sua maioria mulheres, com o relacionamento de filha e esposa. Foi detectada correlação negativa e estatisticamente significante (rs= -.160, $\mathrm{p}=0.01$ ) entre a sobrecarga e as competências do cuidar. Discussão. O perfil dos cuidadores 
deste estudo concorda com a literatura internacional. A correlação entre as variáveis de pesquisa foi documentada em poucos estudos e, portanto, representa um avanço no conhecimento na área de cuidadores. Conclusão. Evidenciou-se uma correlação que pode ser interpretada da seguinte forma: "quanto maior a sobrecarga, menor a competência do cuidar". Achado que evidencia a necessidade de conhecimentos e habilidades para exercer de maneira ideal o papel de cuidador e, por tanto, ter a capacidade de enfrentar situações de sobrecarga.

Palavras-chave:

Cuidador; Doenças crônicas; Pacientes; Adulto; Diabetes Mellitus; Hipertensão.

\section{Introducción}

El aumento de la esperanza de vida ha originado cambios importantes en la pirámide poblacional y con ello el desarrollo de enfermedades crónicas, causantes de 40 millones de muertes a nivel mundial (1). En México, 8.6 millones de personas padecen diabetes, 15.2 millones de personas tienen hipertensión y aproximadamente 18 millones de personas padecen algún tipo de cáncer. Específicamente, el estado de Tamaulipas es una de las cinco entidades federativas de mayor porcentaje con diagnóstico médico de diabetes $(2,3)$.

El desarrollo de estas enfermedades, además de afectar directamente a las personas que las padecen, también repercute en la familia, ya que la evolución de la enfermedad crónica y sus complicaciones implican un aumento en la necesidad de recibir cuidados de alguna persona del entorno familiar, quien se convierte en Cuidador Informal (CI), caracterizado por ser la persona encargada de ayudar en las necesidades básicas instrumentales de la vida diaria del paciente durante la mayor parte del día, sin recibir retribución económica ni un entrenamiento formal para desarrollar su labor $(4,5)$.

Diversos estudios refieren que los CI son mayormente mujeres, con edad entre los 40 y 73 años, escolaridad primaria/secundaria (medio superior) y parentesco de esposa(o) o hija(o), con un tiempo promedio del desempeño del rol de cuidador entre 6.5 y 8 años; además, se destaca que el $43.5 \%$ no cuenta con apoyo de otra persona para el cuidado del enfermo (6-13). Cabe señalar que el rol de CI implica enfrentarse a situaciones críticas relacionadas con el estrés, agotamiento, disminución de oportunidades, motivación y relaciones sociales, así como la posibilidad de presentar síntomas depresivos y ansiedad, problemas de salud y deterioro de la calidad de vida, lo cual puede repercutir en la salud de la díada cuidador-cuidado $(10,14,15)$.

Estudios realizados en cuidadores informales de personas con enfermedades crónicas y dependencia han mostrado diferencias en cuanto a los niveles de sobrecarga, donde se destacan los porcentajes de sobrecarga intensa que van desde el 33 al $85.6 \%$; en el segundo, de $62 \%$, y el tercero, de 33\% $(12,16,17)$. Así mismo, se documentó que, a menor grado de dependencia funcional de la persona cuidada, menor nivel de sobrecarga subjetiva (12). En contraparte, otro estudio realizado en cuidadores de personas con enfermedades crónicas en un hospital de alta complejidad reportó que el $70.7 \%$ no presentó sobrecarga, el $13.4 \%$ presentó sobrecarga leve y el $15.7 \%$ sobrecarga intensa (18).

Aunado a lo anterior, diversos estudios han demostrado que existen factores relacionados al rol de cuidador informal que repercuten directamente en el desarrollo de sobrecarga del cuidado, tales como el sexo, la edad, el nivel de estudios, la actividad laboral, el apoyo de otros cuidadores informales, padecer alguna enfermedad, el tiempo que ha ejercido el rol de cuidador, el parentesco con la persona enferma, la complejidad de las necesidades de la persona enferma y el tiempo de evolución de la enfermedad crónica $(8,18-21)$. Un estudio realizado en cuidadores de personas con enfermedades crónicas identificó una correlación negativa y estadísticamente significativa entre el tiempo que lleva la persona con la enfermedad y la sobrecarga (21).

Un aspecto por destacar es que el desempeño del rol de CI conlleva responsabilidad, compromiso y moral, por lo que se ha demostrado que requiere de competencias del cuidar, las cuales se definen como las capacidades, habilidades y preparación para ejercer la labor de cuidado en el hogar (22). Estas competencias pueden diferir según el tipo de enfermedad crónica, el tiempo y dedicación al cuidado, ya que pueden relacionarse con el desarrollo de experiencia y habilidades para ejercer el rol de cuidador y el parentesco con la persona enferma (23). Se ha documentado que los cuidadores de personas con enfermedades crónicas mostraron tener un nivel medio (40.7\%) y alto $(40.5 \%)$ de competencias del cuidar (24). Sin embargo, en un estudio similar se identificó que el $89.3 \%$ de los cuidadores presentó nivel alto de competencias del cuidar en la escala global 
y por dimensiones, no obstante, dimensiones como conocimiento, unicidad e instrumental registraron niveles medios que generan un área de oportunidad importante (25). Así mismo, cuidadores de personas con enfermedades de mayor demanda de cuidados como el cáncer y falla cardiaca reportaron mayormente nivel medio de competencias del cuidar (37\% a $79.7 \%$ ) $(13,23,26)$.

En un estudio realizado en CI de niños con cáncer, se encontró una correlación significativa entre la dimensión de competencias del cuidar de anticipación con la sobrecarga del cuidador (13). Considerando que el rol CI tiene diversas consecuencias para la salud física, mental y emocional, y que es importante entender la interacción de variables asociadas a ese rol y sus consecuencias, el presente estudio tiene el objetivo de identificar la relación entre las competencias del cuidar y la sobrecarga en cuidadores informales de personas con enfermedades crónicas.

\section{Metodología}

Estudio transversal-correlacional, con muestreo no probabilístico, intencional. La muestra estuvo integrada por 259 cuidadores informales de personas con enfermedades crónicas usuarios de dos Hospitales Generales de Tampico-Tamaulipas. Los criterios de inclusión fueron: ser el cuidador principal de la persona con enfermedad crónica, tener mínimo tres meses ejerciendo el rol de cuidador, no recibir remuneración económica, no tener estudios o capacitación relacionada a la salud y otorgar la autorización y firma del consentimiento informado. Como criterios de exclusión se consideró ser solo acompañante o cuidador remunerado de la persona con enfermedad crónica y no tener habilidades de lectura y escritura, ya que esto interfería en la aplicación del instrumento de medición, cuidadores con discapacidad visual, auditiva o cognitiva. Es importante señalar que esta investigación es derivada del Proyecto "Red de Automanejo de Enfermedades Crónicas".

Los datos sociodemográficos se obtuvieron a través de una cédula de datos sociodemográficos, integrada por dos secciones. La primera incluía ítems sobre la persona con enfermedades crónicas, como sexo, edad, estado civil, escolaridad, ocupación, diagnóstico de la primera enfermedad crónica y tiempo transcurrido desde el diagnóstico de la enfermedad crónica. En la segunda sección se incluyeron ítems relacionados al CI como edad, sexo, ocupación, situación laboral actual, tiempo dedicado al cuidado en meses, parentesco con la persona que recibe el cuidado y, de igual manera, apoyo de otros para brindar el cuidado y la percepción que tiene el CI sobre su salud.

Con el objetivo de evaluar las competencias del cuidar se utilizó el instrumento CUIDAR, con un alfa de Cronbach de 0.92. El instrumento se conforma por 20 ítems, los cuales se agrupan en dimensiones: Conocimiento, Unicidad, Instrumental, Disfrutar, Anticipación y Relación Social. Cada ítem tiene cuatro opciones de respuesta tipo Likert de 0 a 3, que van de 0 (Casi nunca o nunca), 1 (Pocas veces), 2 (Muchas veces) y 3 (Casi siempre o siempre). El instrumento se evalúa por puntos de corte, que reflejan los niveles de competencias del cuidar, los cuales son de 0 a 36 nivel bajo, 37 a 48 nivel medio y 49 a 60 nivel alto de competencias del cuidar, el cual ha reportado una confiabilidad en alfa de Cronbach de 0.92 (22).

Para evaluar la sobrecarga se utilizó la Entrevista de Carga del Cuidador de Zarit (ECCZ), validada en México en 2008, con un alfa de Cronbach de 0.90. El instrumento cuenta con 22 ítems, y una escala de respuesta tipo Likert de 0 a 4 puntos. Su interpretación es mediante las siguientes categorías: de 0 a 47 sin sobrecarga, 48 a 55 sobrecarga leve y 56 a 88 sobrecarga intensa (27).

El procedimiento de recolección de datos se apegó en todo momento a la Ley General de Salud de México en materia de investigación para la salud. Se obtuvieron las autorizaciones de ambas instituciones de salud y posteriormente se acudió a los hospitales, donde los integrantes del equipo realizaron la invitación a los cuidadores familiares para participar en la investigación de forma voluntaria. Una vez que se firmó el consentimiento, se aplicaron los instrumentos de medición. Los datos obtenidos fueron procesados y analizados mediante estadística descriptiva e inferencial con el paquete estadístico Statistical Package the Social Sciences (SPSS) versión 22 para Windows.

\section{Resultados}

Respecto a los datos sociodemográficos de las personas con enfermedades crónicas, se observó que en su mayoría fueron mujeres $(58.3 \%)$, la edad de los usuarios se ubicó entre 18 años y 96 años $(X=67$ años $\mathrm{DE}=15.1)$. Referente al estado civil se identificó que el $48.6 \%$ eran casados, y en cuanto a la ocupación se reportó que el $31.3 \%$ era ama de casa y el $14.7 \%$ era jubilado o pensionado; la escolaridad media fue de 8.2 años (nivel medio superior). La primera enfermedad diagnosticada en los usuarios fue diabetes con el $40.5 \%$ 
de los casos, $27.4 \%$ hipertensión, $10.8 \%$ insuficiencia renal, $6.2 \%$ cáncer y el $15.1 \%$ se agruparon en una categoría que incluía diversas enfermedades neurológicas, respiratorias y mentales. El diagnóstico para estas enfermedades se realizó entre 1 y 60 años atrás $(\underline{X}=14.64$ años, $\mathrm{DE}=12.1)$.

Con respecto a los datos sociodemográficos del cuidador informal, se observó mayor predominio de mujeres $(82.6 \%)$. La edad se ubicó entre 18 y 87 años $(\underline{X}=49$ años, $\mathrm{DE}=12.5)$, de los cuales $77.7 \%$ fueron adultos y $22.3 \%$ adultos mayores. Del mismo modo, se reportó que el $69.1 \%$ de los usuarios eran casados, $44.8 \%$ ama de casa, $21.2 \%$ empleado y escolaridad media de 11.3 años (nivel medio superior) (Tabla 1). Así mismo, se identificó que el tiempo promedio dedicado al cuidado de su familiar es de 80.6 meses, lo que equivale a 6.7 años aproximadamente. Se identificó también que el parentesco que tiene el cuidador con la persona enferma es mayormente esposa (21.2\%) e hija (37.8\%). El 48.6\% de los cuidadores trabaja actualmente y el $49 \%$ no cuenta con un trabajo. De igual manera, el 8.1\% reportó dejar totalmente el trabajo y el $25.1 \%$ parcialmente. El 55.6\% indicó tener el apoyo de otra persona para el cuidado de su familiar, siendo estos mayormente miembros del círculo familiar. Referente a la percepción sobre su salud, el 3.9\% de los cuidadores informales refirió tener problemas de salud (mala salud), el $38.4 \%$ salud regular, el $35.7 \%$ salud buena, el $13.6 \%$ salud muy buena y el $8.4 \%$ salud excelente.

Tabla 1. Características sociodemográficas del cuidador informal de personas con enfermedades crónicas

\begin{tabular}{|c|c|c|c|c|c|}
\hline Variable & $f$ & $\%$ & Variable & $f$ & $\%$ \\
\hline Sexo & \multicolumn{5}{|c|}{ Parentesco con el enfermo } \\
\hline Mujer & 214 & 82.6 & Esposa/compañera & 55 & 21.2 \\
\hline Hombre & 45 & 17.4 & Esposo/compañero & 16 & 6.2 \\
\hline Estado Civil & & & Madre & 16 & 6.2 \\
\hline Casado & 179 & 69.1 & Hija & 98 & 37.8 \\
\hline Soltero & 18 & 6.9 & Hijo & 23 & 8.9 \\
\hline Divorciado & 51 & 19.7 & Hermana & 14 & 5.4 \\
\hline Viudo & 11 & 4.2 & Hermano & 3 & 1.2 \\
\hline Ocupación & & & Nuera & 12 & 4.6 \\
\hline Hogar & 114 & 44.8 & Yerno & 5 & 1.9 \\
\hline Pensionado/Jubilado & 22 & 8.5 & Vecina & 2 & 0.8 \\
\hline Empleado & 55 & 21.2 & Amiga & 2 & 0.8 \\
\hline Comerciante & 15 & 5.8 & Otro & 12 & 4.6 \\
\hline Desempleado & 2 & 0.8 & & & \\
\hline Profesionista & 33 & 12.7 & & & \\
\hline Obrero & 6 & 2.3 & & & \\
\hline
\end{tabular}

Fuente: Cédula de datos sociodemográfico 
En la Figura 1 se presentan los resultados globales y por dimensiones de la variable competencias del cuidar. Referente a las competencias globales, se reportó que el $53.8 \%$ de los CI presentó nivel alto, el 34.3\% nivel medio y el $12 \%$ nivel bajo. En cuanto a las dimensiones, todas presentan un nivel alto de competencias del cuidar en más del $50 \%$ de la muestra; sin embargo, se destaca que las dimensiones de unicidad, instrumental y disfrutar son las que mantienen mayor porcentaje de nivel bajo de competencias.

Figura 1. Niveles de competencias del cuidar total y dimensiones del cuidador informal de personas con enfermedades crónicas

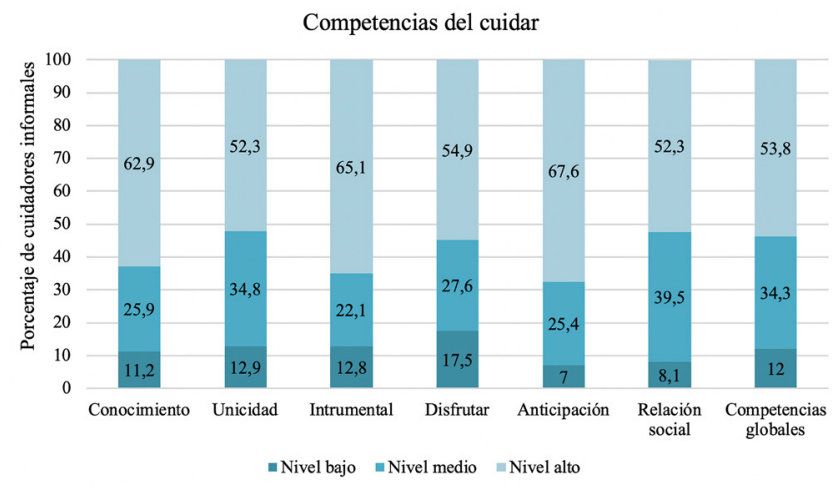

Fuente: Instrumento CUIDAR

Por su parte en la valoración de la sobrecarga, se reportó mayormente la ausencia de este problema, sin embargo, el $12.2 \%$ de los cuidadores presentó sobrecarga de nivel leve a intensa (Figura 2).

Figura 2. Niveles de sobrecarga en cuidadores informales de personas con enfermedades crónicas.

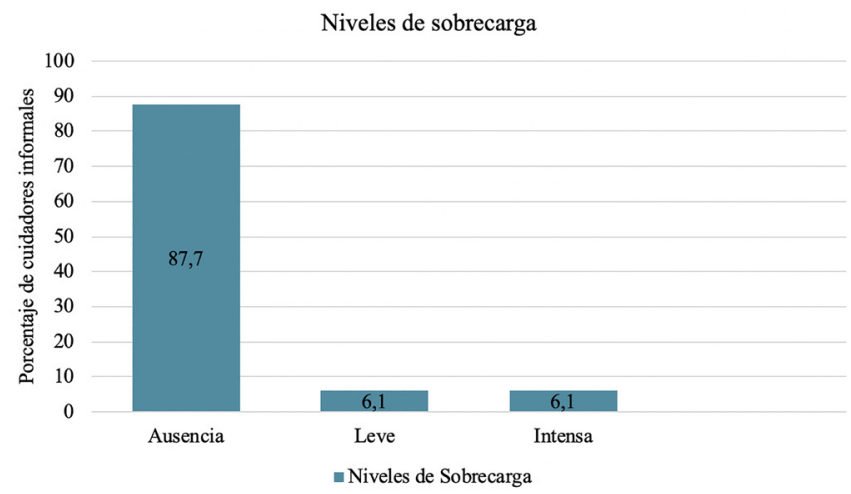

Fuente: Instrumento Zarit
Con el objetivo de identificar la relación entre competencias del cuidar y sobrecarga en cuidadores informales de personas con enfermedades crónicas, se aplicó un coeficiente de correlación Rho de Spearman en el cual se detectó una correlación negativa y estadísticamente significativa ( $\mathrm{p}=0.01)$, lo cual indica que a menor competencias del cuidar mayor nivel de sobrecarga o viceversa (Tabla 2).

Tabla 2. : Correlación entre las competencias del cuidar y sobrecarga en cuidadores informales de personas con enfermedades crónicas.

\section{Nivel Competencias del cuidar}

Spearman Sobrecarga $-0.160 *$

Sig. (bilateral) .0013

*. La correlación es significativa en el nivel 0.05 (2 colas).

\section{Discusión}

Los datos sociodemográficos demostraron que la mayoría de la muestra de los cuidadores informales se conformó por mujeres $(83.6 \%)$, datos que coinciden con otros estudios antecedentes, en los cuales se encontró que entre el $78 \%$ y el $88 \%$ de los CI fueron mujeres (6-11). Referente al parentesco entre el cuidador informal y la persona enferma se identificó que la mayoría fue hija o esposa, datos que concuerdan con diferentes estudios $(6,8,12)$. El sexo y parentesco podrían explicarse con base en lo que sugieren otros autores respecto a la estructura sociocultural de las familias, donde las mujeres se convierten en las responsables del cuidado en el hogar (13).

Con respecto a la edad de los cuidadores informales, se obtuvo que la edad promedio fue de 49 años, dato que coincide con otros estudios realizados en México, Colombia y Cuba (10-12). Estos datos confirman que son los adultos quienes desempeñan el rol de cuidador informal y se puede asumir que la edad del cuidador puede variar según la edad de la persona enferma y el parentesco que exista entre la díada cuidador-cuidado. La escolaridad de los cuidadores informales tuvo una media de 11.3 años (escolaridad media), resultado similar a otros estudios que reportaron educación secundaria y media superior $(7-9,11)$, lo anterior implica el considerar un escenario funcional para fortalecer el conocimiento y formación del cuidador, ya que se infiere que tienen capacidad de aprendizaje y pueden 
ejercer o adquirir habilidades para el cuidado, la toma de decisiones, además pueden contribuir a la seguridad y calidad de vida de la persona enferma.

Así también, se identificó una media de 6.7 años del tiempo dedicado al cuidado de la persona con enfermedad crónica, esto coincide con otros estudios que reportaron un promedio del tiempo dedicado al cuidado de la persona enferma de 6.5 a 8 años $(10,12)$. Las similitudes encontradas respecto al tiempo dedicado al cuidado de un familiar con enfermedad crónica podrían relacionarse a que los CI mantienen un lazo de parentesco familiar y emocional con la persona que se cuida, por lo tanto, el cuidar a un familiar enfermo se convierte en un compromiso moral de tiempo indefinido (12).

Con respecto a la salud percibida de los cuidadores, la categoría con mayor porcentaje $(38.4 \%)$ refirió tener salud regular. A pesar de ser el resultado con mayor porcentaje, difiere con lo reportado en otro estudio similar, ya que el $67 \%$ de los CI indicó tener salud regular (6). Este resultado puede atribuirse a que el cuidador se enfrenta a situaciones críticas derivadas del cuidado brindado a la persona con enfermedad crónica, ya que el estrés, el agotamiento, la falta de apoyo de otros miembros de la familia, tener problemas de salud, presentar síntomas depresivos o ansiedad representan algunos de los múltiples factores que podrían repercutir en la percepción de la salud del CI $(10,14,15)$.

Las competencias del cuidar se identificaron en un nivel mayormente alto en la escala global y por dimensiones, datos que coinciden con otro estudio realizado en muestras similares (24). Sin embargo, se identificaron dimensiones como conocimiento, unicidad e instrumental que presentan áreas de oportunidad y podrían mejorarse a través de intervenciones o programas enfocadas a $\mathrm{CI}(25)$. Otro estudio realizado en CI de niños y adultos con cáncer encontró mayormente nivel medio de competencias del cuidar $(13,23)$, mientras que en CI de personas con falla cardiaca reportaron nivel medio (37\%) y bajo (24\%) (26). Lo anterior pudiera ser explicado por el tipo de enfermedad que padece la persona a cargo del cuidador, ya que la evolución y cronicidad de las diferentes enfermedades crónicas puede diferir según el tipo de enfermedad; por ejemplo, el cáncer y falla cardiaca son enfermedades más agresivas y complejas que generan dependencia y demanda de cuidados. Aunado a ello, el tiempo y dedicación que el CI desempeña podrían favorecer en el desarrollo de experiencia y habilidades (23).
Referente a la sobrecarga se identificó que el $87.8 \%$ de los CI no presentó sobrecarga, sin embargo, el $12.2 \%$ presentó sobrecarga leve a moderada, datos que coinciden con un estudio realizado en cuidadores de personas con enfermedades crónicas en un hospital de alta complejidad (18). No obstante lo anterior, otros estudios reportaron mayormente sobrecarga intensa, dichas diferencias pueden atribuirse a diversos factores como la dependencia de la persona con enfermedad crónica, ya que se ha evidenciado que a menor grado de dependencia menor es el nivel de sobrecarga. Así mismo, se considera el tiempo de cuidado, el apoyo percibido, la relación familiar y emocional entre la díada cuidador-cuidado, variables que podrían generar una "carga" sociocultural de compromiso y deber moral de cuidar al familiar enfermo, lo cual puede repercutir en la percepción de sobrecarga $(13,18)$.

El hallazgo de la correlación negativa y estadísticamente significativa permite inferir que "a mayor competencia del cuidar menor sobrecarga", dicho resultado no se logró contrastar con poblaciones similares, ya que son escasos los estudios que abordan la relación entre estas variables; sin embargo, un estudio realizado en CI de niños con cáncer, mostró ausencia en la correlación entre las competencias del cuidar global y sobrecarga, aunque se identificó una correlación significativa entre la dimensión de anticipación y sobrecarga (13). Las diferencias encontradas pueden derivarse de que el estudio antes citado se realizó exclusivamente en CI de niños con cáncer, por lo que el CI suele ser la madre o padre del niño, parentesco que genera un vínculo emocional importante que podría repercutir en la percepción de la sobrecarga. Aunado a lo anterior, es importante destacar que las enfermedades crónicas incluidas en el presente estudio, tales como hipertensión y diabetes, tienen un proceso de deterioro y evolución diferente, datos que confirman lo antes señalado por distintos autores referente a que la sobrecarga y las competencias del cuidar pueden diferir según el tipo de enfermedad y deterioro generado de la misma $(17,23)$.

El hallazgo de la correlación entre competencias del cuidar y sobrecarga, también podría encontrar explicación en estudios antecedentes que refieren que el tiempo prolongado en el desempeño en rol de cuidar puede desarrollar deterioro físico, mental, problemas económicos (28), síntomas depresivos y de ansiedad (10), que se traducen en sobrecarga del cuidado, problema que repercute directamente en las capacidades, habilidades y preparación para ejercer la labor de cuidado en el hogar. 


\section{Conclusión}

Los resultados permitieron cumplir el objetivo de estudio e identificar una correlación negativa entre las competencias del cuidar y la sobrecarga del CI, que se puede interpretar "a menor competencias del cuidar mayor nivel de sobrecarga". Estos hallazgos evidencian que el CI requiere de diversas necesidades en conocimientos y habilidades para ejercer de forma óptima el rol de CI y por ende, tener la capacidad de afrontar situaciones de sobrecarga. Así también, a partir de los datos sociodemográficos se concluye que el perfil de cuidadores concuerda en que son mujeres con edad promedio de 49 años, quienes tienen en su mayoría escolaridad media y parentesco de esposas o hijas de las personas que padecen alguna enfermedad crónica.

Por lo tanto, los resultados de este estudio aportan conocimiento para generar estrategias educativas de enfermería para aumentar las competencias del cuidar y con ello disminuir la sobrecarga de los CI y el riesgo de desarrollar enfermedades en cuidadores informales, así como prevenir complicaciones en las personas que están a su cuidado.

Finalmente, se recomienda implementar estudios en muestras de cuidadores de personas con diferentes enfermedades crónicas en etapas avanzadas, ya que estas pueden generar mayor dependencia y necesidad de cuidados, así como estudios que analicen la relación entre la sobrecarga, las competencias y otras variables como dependencia. Así también, se recomienda implementar estudios con muestras probabilísticas para poder realizar inferencias a nivel de población.

\section{Referencias}

1. Organización Mundial de la Salud. Enfermedades no transmisibles. Nota Descriptiva. Junio 2018 [Internet]. Washington DC: OMS; 2018 [citado 19 de mayo 2020]. Disponible en https://www.who.int/es/newsroom/factsheets/detail/noncommunicable-diseases

2. Encuesta Nacional de Salud y Nutrición 2018. Presentación de resultados [Internet]. Ciudad de México; 2019. Recuperado de: https://ensanut.insp.mx/encuestas/ensanut2018/doctos/informes/ensanut_2018 presentacion_resultados.pdf

3. Instituto nacional de Cancerología México. El cáncer en el mundo y México [Internet]. INCAN. 2019. [accesado 18 de mayo 2020]. Disponible en: http://www. infocancer.org. $\mathrm{mx} / \mathrm{c}=$ conocer-el-cancer\&a $=$ estadisticas-mundiales-y-locales

4. Jofré AV, Sanhueza AO. Evaluación de la sobrecarga de cuidadoras/es informales. Ciencia y enfermería.
2010;16(3):111-120. Doi: https://doi.org/10.4067/ $\underline{\mathrm{S} 0717-95532010000300012}$

5. López GE. Cuidar a los que cuidan: los cuidadores informales. Revista Uruguaya de Enfermería. 2016;11(2):49-58. Recuperado de: http://rue.fenf. edu.uy/index.php/rue/article/view/198

6. Villegas-Ceja MH, Aguilar-Chagoyán MA, Espericueta-Medina M, Medina-Sánchez M, Luján-López ME. Desempeño del rol de cuidador primario y eficiencia del programa Atención Hospitalaria a Domicilio. Revista Enfermería Instituto Mexicano Seguro Social. 2014;22(1):25-32. Disponible en: https://www.medigraphic.com/cgi-bin/new/resumen.cgi?IDARTICULO $=47961$

7. Andreotti DN, De Souza MB, Neri KH, Golghetto CF, Correa FL, De Oliveira GL, Martins GA. Comparative study between formal and informal caregivers of older adults. Ciência \& Saudé Coletiva. 2018;23(11):3789-3798. Doi: https://doi. org/10.1590/1413-812320182311.16932016

8. Peña-Ibáñez F, Álvarez-Ramírez M, Melero-Martín J. Sobrecarga del cuidador informal de pacientes inmovilizados en una zona de salud urbana. Enfermería Global. 2016;15(43):100-111. Disponible en: http://scielo.isciii.es/scielo.php?script=sci abstract\&pid=S169561412016000300004

9. Martínez RL. Ansiedad, depresión y sobrecarga en cuidadores de adultos centenarios. Revista Cubana Salud Pública. 2018;44(4):61-72. Disponible en: http://scielo.sld.cu/ scielo.php?script=sci abstract\&pid=S086434662018000400061\&lng=es\&nr$\underline{m}=$ iso

10. Palomé G, Gallegos R, Xeque A, Juárez A, Perea M. Nivel de sobrecarga en el cuidador principal del paciente crónico hospitalizado. Digital CIENCIA@uaqro. 2014;1(1):1-10. Disponible en: https://www.uaq.mx/investigacion/revista_ciencia@, uaq/ArchivosPDF/ v7-n1/03Articulo.pdf

11. Ávila-Toscano JH, Vergara-Mercado M. Calidad de vida en cuidadores informales de personas con enfermedades crónicas. Aquichan. 2014;14(3):417429. Doi: https://doi.org/10.5294/aqui.2014.14.3.11 12. De Valle-Alonso MJ, Hernández-López IE, Zúñiga-Vargas ML, Martínez-Aguilera P. Sobrecarga y Burnout en cuidadores informales del adulto mayor. Enfermería Universitaria. 2015; 12(1):19-27. Doi: https://doi.org/10.1016/j.reu.2015.05.004

13. Carreño S, Arias M. Competencia para cuidar en el hogar y sobrecarga en el cuidador del niño con cáncer. Gaceta Mexicana de Oncología. 2016;15(6):336-343. https://doi.org/10.1016/j.gamo.2016.09.009

14. Ramírez-Perdomo CA, Chacón-Cuellar E, Romero -Perdomo AY. Enfrentar la dureza de la enfermedad crónica, retos de la díada: cuidador/persona cuidada. Cultura de los Cuidados. 2018;22(52):46-57. Disponible 
en: http://dx.doi.org/10.14198/cuid. 2018.52.04

15. Abellán-Hervás MJ, López-Fernández C, Santi-Cano MJ, Deudero-Sánchez M., Picardo-García JM. ¿Quién es el receptor de intervenciones sociosanitarias y cuáles son sus necesidades? Enfermería Clínica. 2016;26(1):49-54. Disponible en: https:// doi.org/10.1016/j.enfcli.2015.08.004

16. Rodríguez-Medina RM, Landeros-Pérez M. Sobrecarga del agente de cuidado dependiente y su relación con la dependencia funcional del adulto mayor. Enfermería Universitaria. 2014;11(3):8793. Disponible en: http://www.revistas.unam.mx/ index. php/reu/article/ view/47585/42809

17. Rodríguez-González A, Rodríguez-Míguez E, Duarte-Pérez A, Díaz-Sanisidro E, Barbosa-Álvarez A, Clavería A. Grupo Zarit. Estudio observacional transversal de la sobrecarga en cuidadoras informales y en los determinantes relacionados con la atención a las personas dependientes. Atención Primaria. 2017;49(3):156-165. Doi: https://doi.org/10.1016/j.aprim.2016.05.006

18. Cantillo-Medina CP, Ramírez-Perdomo CA, Perdomo-Romero AY. Habilidad de cuidado en cuidadores familiares de personas con enfermedad crónica y sobrecarga percibida. Ciencia y enfermería. 2018;24(16):1-12. Disponible en: http://dx.doi. org/10.4067/s0717-95532018000100216

19. Cerquera A, Galvis A. Efectos de cuidar personas con Alzheimer: un estudio sobre cuidadores formales e informales. Pensamiento psicológico. 2014;12(1):149-167. https://doi.org/10.11144/Javerianacali.PPSI12-1.ecpa

20. Piñeiro I, Rodríguez S, Albite A, Freire C, Ferradás M. Sobrecarga y salud percibida en cuidadores informales de pacientes con enfermedad mental. European Journal of Health Research. 2017;3(3):185196. doi: 10.30552/ejhr.v3i3.75

21. Carreño SP, Chaparro-Díaz L. Agrupaciones de cuidadores familiares en Colombia: perfil, habilidad de cuidado y sobrecarga. Pensamiento Psicológico. 2017;15(1):87-101. http://dx.doi.org/10.11144/Javerianacali.PPSI15-1.ACFC

22. Carrillo-González G, Sánchez-Herrera B, Vargas-Rosero E. Desarrollo y pruebas psicométricas del Instrumento "cuidar" - versión corta para medir la competencia de cuidado en el hogar. Revista de la Universidad Industrial de Santander. 2016;48(2):222-231. doi: http://dx.doi. org/10.18273/revsal.v48n2-2016007

23. Carrillo GM, Carreño SP, Sánchez LM. Competencia para el cuidado en el hogar y carga en cuidadores familiares de adultos y niños con cáncer. Revista Investigaciones Andina. 2018;20(36):87-101. Disponible en: https://doi.org/10.33132/01248146.971

24. Carrillo-González G, Barreto-Osorio R, Arboleda
L, Gutiérrez-Lesmes O, Melo B, Ortiz V. Competencia para cuidar en el hogar de personas con enfermedad crónica y sus cuidadores en Colombia. Revista Facultad de Medicina. 2015;63(4):668675. doi: http://dx.doi.org/10.15446/revfacmed. v63. n4.50322

25. Vega AO, González ED. Soporte social y competencia para el cuidado en cuidadores de personas con enfermedad crónica en la provincia de Ocaña. En XVI Coloquio Panamericano de Investigación en Enfermería. 2018. Disponible en: http://www. coloquioenfermeria2018.sld.cu/index.php/coloquio/2018/paper/view/566/474

26. Achury D, Restrepo A, Torres N, Buitrago A, Neira N, Devia P. Competencia de los cuidadores familiares para cuidar a los pacientes con falla cardiaca. Revista Cuidarte. 2017;8(3):1721-1732.

27. Alpuche V, Ramos B, Rojas M, Figueroa C. Validez de la entrevista de carga de Zarit en una muestra de cuidadores primarios informales. Psicología y Salud. 2008;18(2):237-245.

28. Navarro-Sandoval C, Uriostegui-Espíritu L, Delgado-Quiñones E, Sahagún-Cuevas M. Depresión y sobrecarga en cuidadores primarios de pacientes geriátricos con dependencia física de la UMF 171, Revista Médica del Instituto Mexicano del Seguro Social. 2014;55(1):25-31. Disponible en: https://www.medigraphic.com/cgi-bin/new/resumen. cgi?IDARTICULO=71837 